\title{
Use of biofunctional prosthetic system following partial maxillary resection: a clinical report
}

\author{
A. NEKORA-AZAK, G. EVLIOGLU, M. ÖZDEMIR-KARATAS, \& H. KESKIN Department of \\ Maxillofacial Prosthodontics, Faculty of Dentistry, University of Istanbul, Istanbul, Turkey
}

SUMMARY This clinical report describes the prosthodontic rehabilitation of an edentulous patient with a maxillary defect using the biofunctional prosthetic system. The Biofunctional Prosthetic System brand denture includes a comprehensive technique for impressions, record taking, tooth placement, fabrication and processing. The advantage of this technique is to provide patients with optimal form, function, and aesthetics in complete dentures. This system is based on a team effort and systematic approach to removable prosthetic treatment. KEYWORDs: maxillary defect, edentulous patient, prosthetic rehabilitation

\section{Accepted for publication 18 October 2004}

\section{Introduction}

Rehabilitation of the maxillectomy defect has been well defined for prosthodontists and surgeons (1-10). All prosthodontists are aware of the basic objectives of prosthodontic therapy. A comfortable, cosmetically acceptable prosthesis that restores the impaired physiologic activities of speech, deglutition, and mastication is a basic objective of prosthodontic care.

The primary goal of prosthetic obturation is closure of the maxillectomy defect and separation of the oral cavity from the sino-nasal cavities. An unfavourable situation for prosthetic rehabilitation occurs when the size of a defect is so large that it overwhelms the remaining structures that stabilize prosthesis over the defect. Instability of the obturator results in air and fluid leakage through the nasal cavity and thereby compromises function.

The Biofunctional prosthetic system (BPS) is a complete system for the fabrication of dentures, designed to provide patients with optimal form, function, and aesthetics. Based on an advanced and logical concept for the treatment of edentulous patients with a maxillary defect, the system includes a comprehensive technique for impressions, record taking, tooth selection, placement, fabrication and processing. This clinical report describes the use of this type of prosthetic system following partial maxillary resection.

\section{Clinical report}

A 63-year-old woman was referred to the maxillofacial prosthetics clinic at Istanbul University, Faculty of Dentistry by her head and neck surgeon for evaluation for prosthetic treatment. The patient had successfully worn a maxillary complete denture and a mandibular removable partial denture for 7 years. She had a history of squamous cell carcinoma in the right maxilla. Following $3000 \mathrm{rad}$ of radiation therapy, a partial maxillary resection was completed.

The BPS recommends impressions, similar in principle to the mucostatic method, that minimally disturbs or compresses tissues on which a denture base will set, and utilizes a combination of irreversible hydrocolloids of varying densities together in the same impression, to capture tissues in a static state (11). Low density impression material (Syringe Accu-Gel)* was syringed into the vestibular area from the maxillary tuberosity to the defect (Fig. 1). The impression tray, (Tray AccuGel)* which was already loaded with high-density

*Ivoclar-Vivadent, Schaan, Liechtenstein. 


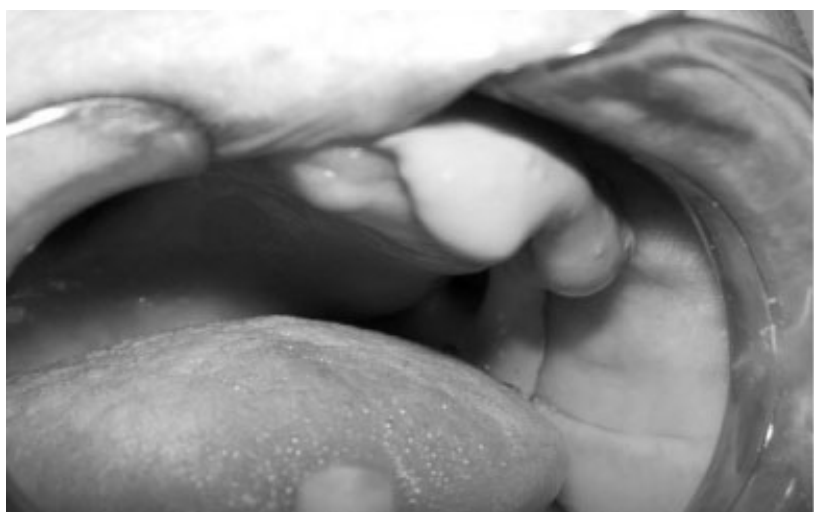

Fig. 1. The light bodied syringe accu-gel has been injected within the entire extend of the maxillary vestibular space.

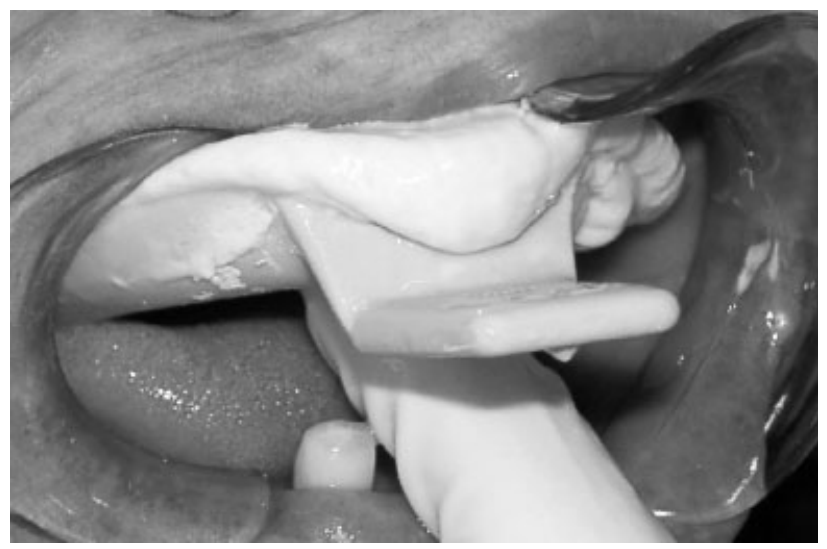

Fig. 2. The impression tray with high-density hydrocolloid was lightly placed into the patient's mouth.

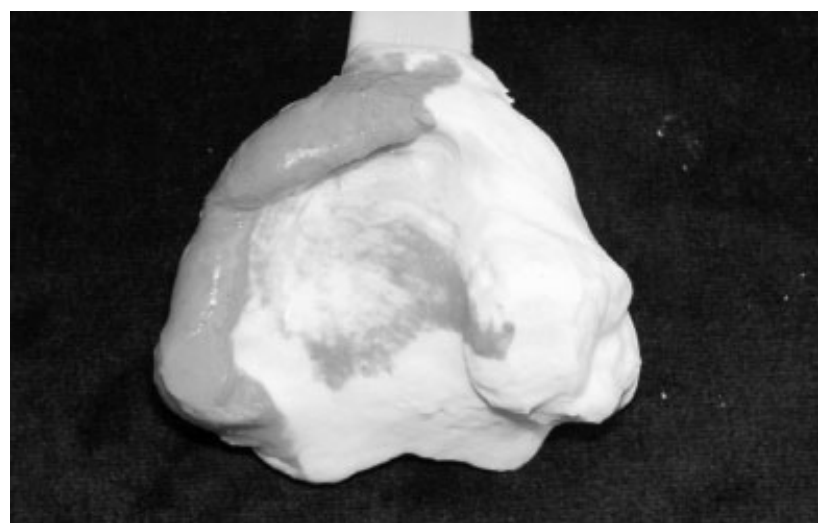

Fig. 3. The completed maxillary impression.

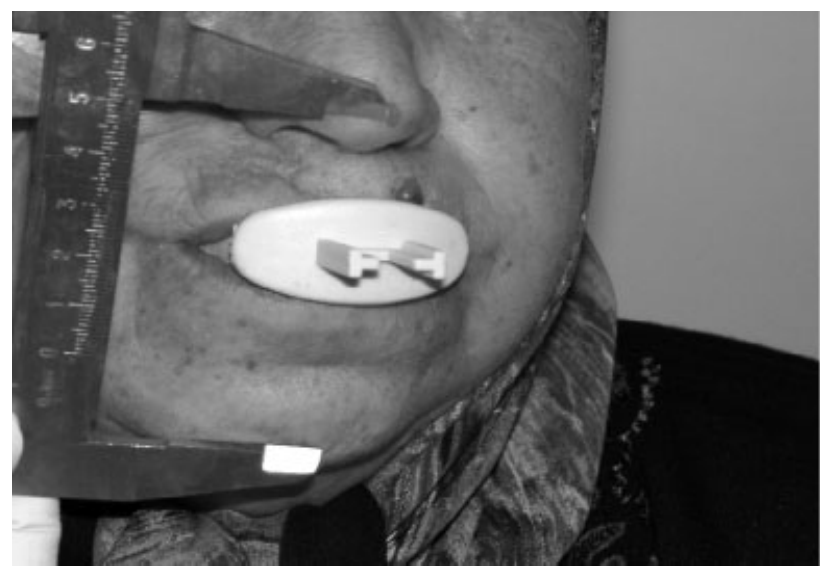

Fig. 4. Centric tray to determine the occlusal position.

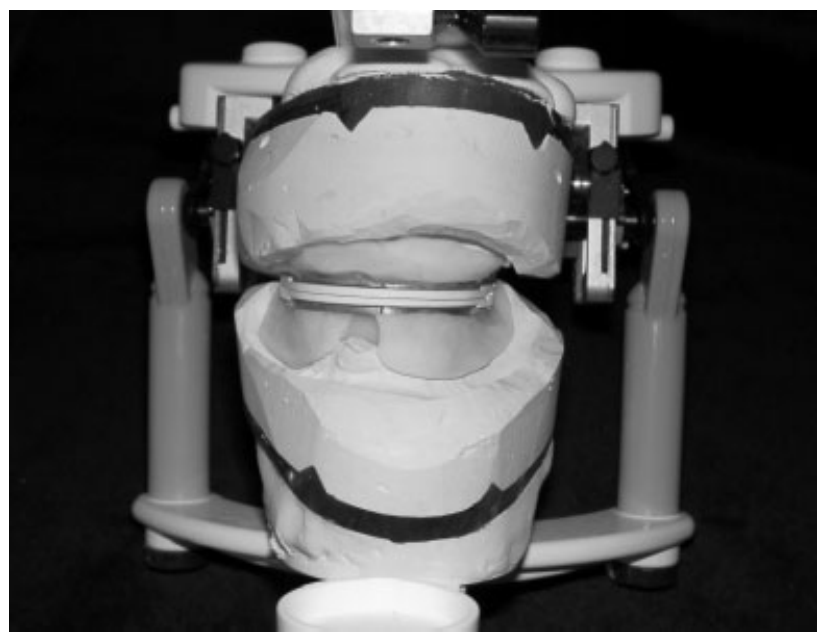

Fig. 5. Proceeding with mounting the primary casts and setting up the Gnathomater registration plates.

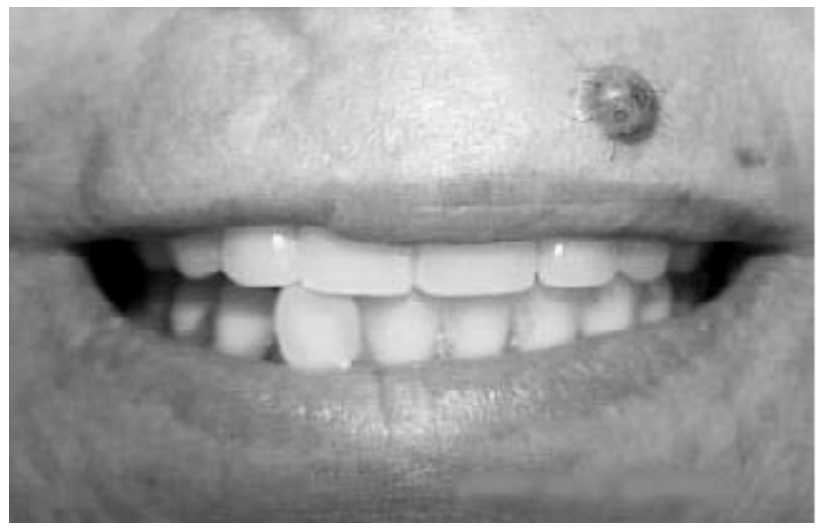

Fig. 6. Final maxillary and mandibular removable prostheses.

(C) 2005 Blackwell Publishing Ltd, Journal of Oral Rehabilitation 32; 693-695 
hydrocolloid was lightly placed into the patient's mouth (Fig. 2). After the hydrocolloid materials had set, the impression tray was gently removed and checked for accuracy (Fig. 3). The Tray Accu-Gel and The Syringe Accu-Gel are different, but chemically compatible irreversible hydrocolloids designed to work together in the mouth. The mandibular impression was taken in the same manner as the maxillary impression.

At the same appointment, the primary impressions were taken and provisional jaw registration was made using the Centric Tray* (Fig. 4). The aim of the Centric Tray is to determine the occlusal position.

All of these allow the laboratory to proceed with mounting the primary casts and setting up the Gnathometer M tracing device*, which facilitates the clinical procedures of second impression taking, face bow record and jaw registration (Fig. 5).

Second impressions with Zinc oxide Eugenol impression paste ${ }^{\dagger}$, jaw registration and try-in was accomplished. The definitive obturator prosthesis with hollow bulb was fabricated using routine laboratory procedures (Fig. 6).

\section{Discussion}

All prosthodontists are aware of the basic objectives of prosthodontic therapy. The basic objectives must also be applied to the patient requiring maxillofacial rehabilitation. Ideally, a patient with an acquired maxillary defect should be provided with an obturator prosthesis that is comfortable, restores adequate speech, deglutition, and mastication, and is acceptable cosmetically. If there is any patient in whom compromised remaining structures must be preserved, it is the maxillofacial patient.

This report describes the prosthetic treatment of an edentulous patient with a maxillary defect using an unconventional prosthetic procedure. The treatment proceeded mainly in accordance to BPS. This system simplified the clinical and laboratory procedures and reduced the number of patient visits and the cost of the prosthesis. The patient is cooperating well and is motivated to maintain a good standard of obturator prosthesis.

\section{References}

1. Minsley GE, Warren DW, Hinton V. Physiologic responses to maxillary resection and subsequent obturation. J Prosthet Dent. 1987;57:338-344.

2. Watson RM, Gray BJ. Assessing effective obturation. J Prosthet Dent. 1985;54:88-93.

3. Shipman B. Evaluation of occlusal force in patients with obturator defects. J Prosthet Dent. 1987;57:81-84.

4. Desjardins RP. Obturator prosthesis design for acquired maxillary defects. J Prosthet Dent. 1978;39:424-435.

5. Beumer J, Curtis T, Marunick M. Maxillofacial rehabilitation: prosthodontic and surgical considerations. St Louis: Ishiyaku EuroAmaerica; 1996:233-237.

6. Aramany MA. Basic principles of obturator design for partially edentulous patients: Part I: classification. J Prosthet Dent. 1978;40:554-557.

7. Aramany MA. Basic principles of obturator design for partially edentulous patients: Part II: design principles. J Prosthet Dent. 1978;40:656-662.

8. King GE, Gay WD. Application of various removable partial denture design concepts to a maxillary obturator prosthesis. J Prosthet Dent. 1979;41:316-318.

9. Parr GR, Tharp GE, Rahn AO. Prosthodontic principles in the framework design of maxillary obturator prostheses. J Prosthet Dent. 1989;62:205-212.

10. Martin JW, King GE. Framework retention for maxillary obturator prostheses. J Prosthet Dent. 1984;51:669-672.

11. Roraff AR, Stansbury BE. Errors caused by dimensional change in mounting materials. J Prosthet Dent. $1972 ; 28: 247-252$.

Correspondence: Dr Aysen Nekora-Azak, Innere Altachen 21, $\mathrm{CH}-4800$ Zofingen, Switzerland.

E-mail: aysenazak@bluewin.ch

\footnotetext{
${ }^{\dagger}$ S.S. White Manufacturing, Gloucester, England.
} 